\title{
ERRATUM
}

Alfio Grillo • Raphael Prohl • Gabriel Wittum

\section{Erratum to: A poroplastic model of structural reorganisation in porous media of biomechanical interest}

Published online: 14 January 2016

(C) Springer-Verlag Berlin Heidelberg 2016

Erratum to: Continuum Mech. Thermodyn.

DOI 10.1007/s00161-015-0465-y

We would like to perform the following corrections to our paper [1].

\section{Correction to Sect. 3}

In Eq. (11b), $I_{1}\left(\boldsymbol{C}_{\mathrm{e}}\right)$ should be replaced with $\hat{I}_{1}\left(\boldsymbol{C}_{\mathrm{e}}\right)$. Thus, the corrected equation reads

$$
I_{2}=\hat{I}_{2}\left(\boldsymbol{C}_{\mathrm{e}}\right)=\frac{1}{2}\left\{\left[\hat{I}_{1}\left(\boldsymbol{C}_{\mathrm{e}}\right)\right]^{2}-\operatorname{tr}\left[\left(\boldsymbol{\eta}^{-1} \boldsymbol{C}_{\mathrm{e}}\right)^{2}\right]\right\}=\frac{1}{2}\left\{I_{1}^{2}-\operatorname{tr}\left[\left(\boldsymbol{B}_{\mathrm{p}} \boldsymbol{C}\right)^{2}\right]\right\},
$$

\section{Corrections to Sect. 4}

1. In the sentence starting one line after Eq. (63c) with "In particular, ...", and ending with Eq. (65), the wording "acoustic tensor" is incorrect and should be replaced with "first elasticity tensor". Accordingly, the corrected text should read as reported below:

"In particular, $\mathbb{A}_{n, k-1, l-1}^{\mathrm{sc}}$ is the constitutive part of the first elasticity tensor computed at the $l$ th iteration in $\boldsymbol{B}_{\mathrm{p} n}$ and at the $k$ th iteration in $p_{n}$ and $\chi_{n}$, i.e.

$$
\mathbb{A}_{n, k-1, l-1}^{\mathrm{sc}}=\frac{\partial \hat{\boldsymbol{P}}_{\mathrm{sc}}}{\partial \boldsymbol{F}}\left(\boldsymbol{F}_{n, k-1}, \boldsymbol{B}_{\mathrm{p} n, l-1}\right),
$$

The online version of the original article can be found under doi:10.1007/s00161-015-0465-y.

A. Grillo $(\bowtie)$

DISMA “G.L. Lagrange”, Politecnico di Torino, C.so Duca degli Abruzzi 24, 10129 Torino, TO, Italy

E-mail: alfio.grillo@polito.it

R. Prohl

Steinbeis Center, Simulation in Technology, Bussardweg 6, 75446 Wiernsheim, Germany

E-mail: raphael@techsim.org

G. Wittum

G-CSC, Goethe Universität Frankfurt, Kettenhofweg 139, 60325 Frankfurt am Main, Germany

E-mail:wittum@gcsc.uni-frankfurt.de 
while $\mathbb{L}_{n, k-1, l-1}$ is a fictitious elasticity tensor, introduced by the algorithm, and induced by the Gâteaux derivative of the functional $\mathfrak{L}$ with respect to the deformation [cf. (58)], i.e.

$$
D_{\chi} \mathfrak{L}\left(\Lambda_{n, k-1, l-1}\right)\left[\boldsymbol{h}_{n, k}\right]:=\int_{\mathcal{C}_{\mathrm{R}}} \boldsymbol{g} \operatorname{Grad} \tilde{\boldsymbol{u}}: \mathbb{L}_{n, k-1, l-1}: \boldsymbol{H}_{n, k} . "
$$

2. In the text starting one line after Eq. (65) with "It is important ..." and ending with "... fails to be satisfied.", the reference to the papers $[3,4]$ is incorrect. Hereby, we reformulated the text and corrected the reference to the works $[3,4]$ as reported below.

"The effective elasticity tensor

$$
\left(\mathbb{A}_{n, k-1, l-1}^{\mathrm{sc}}-\mathbb{L}_{n, k-1, l-1}\right)
$$

should be such that the matrix associated with the bilinear form $a(\cdot, \cdot)$ in the Finite Element discretisation of (62a) is invertible. Although this is actually the case for the problem at hand, we have not formulated theorems yet, which predict when this condition fails to be satisfied. In linearised Elasticity, it is often required that the elasticity tensor is strongly elliptic, a condition that is also related to the existence of 'plane progressive elastic waves in all possible directions' and to the reality of wave speeds [2]. In the context of Elastoplasticity, the issue of strong ellipticity has been studied, for example, in [3], whereas a spectral analysis of the acoustic tensor associated with a particular constitutive law for the stress tensor can be found in [4]."

Note that the references [2,3], and [4] correspond, respectively, to the references [68, 17], and [18] of the original paper [1].

3. In the sentence "The algorithm proposed in this paper [...] with respect to $\boldsymbol{B}_{\mathrm{p} n}$." it should be emphasised that the algorithm requires a linearisation also with respect to the pressure evaluated at the time instant $t_{n}$, i.e. $p_{n}$. Accordingly, the corrected text should read as follows:

"The algorithm proposed in this paper requires a linearisation with respect to $p_{n}$ and $\chi_{n}$, and one with respect to $\boldsymbol{B}_{\mathrm{p} n} . "$

\section{Corrections to Sect. 5}

In the last paragraph, the sentence "Within the Newton iteration, ... linearised sub-problems." should be changed as follows:

"Within the Newton iteration, an ILU-decomposition, accelerated by a Bi-CGSTAB method, solves the linearised sub-problems."

\section{References}

1. Grillo, A., Prohl, R., Wittum, G.: A poroplastic model of structural reorganisation in porous media of biomechanical interest. Continuum Mech. Thermodyn. 28(1-2), 579-601 (2016) doi:10.1007/s00161-015-0465-y

2. Marsden, J.E., Hughes, T.J.R.: Mathematical Foundations of Elasticity. Dover, New York (1983)

3. Bigoni, D., Zaccaria, D.: Strong ellipticity of comparison solids in elastoplasticity with volumetric non-associativity. Int. J. Solids Struct. 29(17), 2123-2136 (1992)

4. Bigoni, D., Zaccaria, D.: On the eigenvalues of the acoustic tensor in elastoplasticity. Eur. J. Mech. A/Solids 13(5), 621638 (1994) 